\title{
Diagnosis and classification of Goodpastures disease (anti-GBM)
}

Thomas Hellmark and Mårten Segelmark

\section{Linköping University Post Print}

\section{Tweet}

N.B.: When citing this work, cite the original article.

Original Publication:

Thomas Hellmark and Mårten Segelmark, Diagnosis and classification of Goodpastures disease (anti-GBM), 2014, Journal of Autoimmunity, (48-49), 108-112.

http://dx.doi.org/10.1016/j.jaut.2014.01.024

Copyright: Elsevier

http://www.elsevier.com/

Postprint available at: Linköping University Electronic Press

http://urn.kb.se/resolve?urn=urn:nbn:se:liu:diva-106524 


\section{Anti-GBM disease (Goodpasture's disease)}

Thomas Hellmark, PhD, Associate professor, corresponding author

Postal address: Department of Nephrology, Clinical Sciences in Lund, BMC-B13, Lund University, 22184 Lund, Sweden

Telephone: +46-46-2220701

Email: Thomas.Hellmark@med.lu.se

Mårten Segelmark, $\mathrm{MD}, \mathrm{PhD}$, Professor

Postal address: Division of Drug Research/Nephrology, Department of Medical and Health Sciences, Linköping University, 58185 Linköping, Sweden

Telephone: +46-10-1032297

E-mail: marten.segelmark@liu.se

\section{Highlights}

- Anti-GBM disease is now a preferred term for what was earlier called Goodpasture's syndrome or Goodpasture's disease

- Anti-GBM disease is now classified as small vessel vasculitis caused by in situ immune complex formation

- The diagnosis relies on the detection of anti-GBM in tissues or circulation in conjunction with alveolar or glomerular disease

- Therapy is effective only when detected at an early stage, making a high degree of awareness necessary to find these rare cases

- 20-35\% have anti-GBM and MPO-ANCA simultaneously, which necessitates testing for anti-GBM whenever acute test for ANCA is ordered in patients with renal disease. 


\section{Abstract}

Goodpasture's disease or anti-glomerular basement membrane disease (anti-GBMdisease) is included among immune complex small vessel vasculitides. The definition of anti-GBM disease is a vasculitis affecting glomerular capillaries, pulmonary capillaries, or both, with GBM deposition of anti-GBM autoantibodies. The disease is a prototype of autoimmune disease, where the patients develop autoantibodies that bind to the basement membranes and activate the classical pathway of the complement system, which start a neutrophil dependent inflammation. The diagnosis of anti-GBM disease relies on the detection of anti-GBM antibodies in conjunction with glomerulonephritis and/or alveolitis. Overt clinical symptoms are most prominent in the glomeruli where the inflammation usually results in a severe rapidly progressive glomerulonephritis. Despite modern treatment less than one third of the patients survive with a preserved kidney function after 6 months follow-up. Frequencies varies from 0,5 -1 cases per million inhabitants per year and there is a strong genetic linkage to HLA-DRB1*1501 and DRB $1 * 1502$.

\section{Keywords}

Anti-GBM disease, Goodpasture's disease, autoantibodies, glomerulonephritis, vasculitis 


\section{1. Background and Definitions}

\subsection{Definitions}

In the most recent version of the Chapel Hill nomenclature of vasculitis the disease entity "Anti-glomerular basement membrane disease" (anti-GBM-disease) is included among immune complex small vessel vasculitides [1]. The definition anti-GBM disease according this document is a vasculitis affecting glomerular capillaries, pulmonary capillaries, or both, with GBM deposition of anti-GBM autoantibodies. Lung involvement causes pulmonary haemorrhage, and renal involvement causes glomerulonephritis with necrosis and crescents. It is noted in the document that the terms anti-GBM disease and anti-GBM-antibodies is somewhat confusing as the antibodies react also with the alveolar basement membrane.

\subsection{History}

The history of what we today call anti-GBM disease dates back to 1958 when Stanton et al reported as series of patients with the combination of glomerulonephritis and lung haemorrhage and introduced the term Goodpasture's syndrome in recognition of a case report from 1919 by E.W. Goodpasture [2, 3]. When the technique for direct immunofluorescence (IF) was introduced in renal medicine it was shown that such patients often had a continuous linear deposition of immunoglobulin $\mathrm{G}$ along their glomerular basement membrane (figure 1). A pathogenic role of these antibodies was demonstrated by elution of autoantibodies from human kidneys and transfer to primates [4]. This led to a tendency to narrow the use of the term Goodpasture's syndrome to include only cases with the triad of lung haemorrhage, renal failure and anti-GBM antibodies. Later when it was shown that many patients with anti-GBM do not have lung haemorrhage and most patients with lung haemorrhage and glomerulonephritis do 
not have anti-GBM, the term Goodpasture's disease was preferred for anti-GBM in conjunction with glomerulonephritis and/or alveolar bleeding.

\section{Pathogenesis}

\subsection{Pathogenesis}

Anti-GBM disease is a prototype of autoimmune disease, where B- and T-cell mediated responses constitute the basis of the pathogenic process. The patients develop autoantibodies against the non-collagenous domain 1 of the $\alpha 3$-chain of type IV collagen ( $\alpha 3(\mathrm{IV}) \mathrm{NC} 1)$ [5]. The $\alpha 3$ (IV) chain is expressed in a few specialized basement membranes in the body such as the GBM, the alveolar basement membrane, basement membranes in the testis, the inner ear, the eye and the choroid plexus [6]. IgG antibodies against $\alpha 3(\mathrm{IV}) \mathrm{NC} 1$ bind to these basement membranes and activate the classical pathway of the complement system, which start a neutrophil dependent inflammation. Overt clinical symptoms are most prominent in the glomeruli where the inflammation usually results in a severe rapidly progressive glomerulonephritis.

Despite modern treatment less than one third of the patients survive with a preserved kidney function after 6 months follow-up [7, 8].

Numerous animal models have been described showing the pathogenic role of the antiGBM antibodies. In a classic experiment, primates developed glomerulonephritis after injection of autoantibodies eluted from the kidneys of a nephrectomised patient suffering from anti-GBM disease [4]. Indirect proof of the pathogenic potential of the antibodies was given by the reappearance of disease in a renal transplant given to a patient with persistent high levels of circulating anti-GBM antibodies. Temporal relationships between relapse and reoccurrence of autoantibodies have also been 
reported [9]. The titre of circulating anti-GBM antibodies, as measured by ELISA, has been shown to have prognostic importance $[7,10]$.

In 1984 it was shown that the anti-GBM antibodies reacted with peptides around 25 and $50 \mathrm{kD}$ and these were later shown to be derived from the non-collagenous domain (NC1) of type IV collagen [5]. The peptides were identified as a new chain of type IV collagen, the $\alpha 3$-chain. It was also shown that the epitope were cryptic and hidden in the NC1 hexamer [11]. Patients have a polyclonal immune response and develop autoantibodies to different parts of the antigen $[12,13]$. Two major epitopes have been identified [14], but only antibodies against one reflect the toxicity of the antibodies [15]. This epitope is situated near the triple helical junction (figure 2). The epitope is a cryptotope and accessibility for the anti-GBM antibodies is normally limited. The cryptic properties have recently been shown to be due to S-hydroxylysyl-methionine and S-lysyl-methionine cross-links, which stabilize the $\alpha 3 \alpha 5$-heterodimers and $\alpha 4 \alpha 4$ homodimers in the $\mathrm{NC} 1$ hexamer domains respectively $[16,17]$. However, it has been shown that oxidants can open up the structure, as can certain subpopulations of antiGBM antibodies [14].

Nonetheless there is emerging evidence for a substantial T-cell component in anti-GBM disease. The autoantibody IgG subclass distribution is compatible with a T-cell mediated reaction towards a protein antigen. A mononuclear interstitial cell infiltrate is invariably seen in human anti-GBM disease, consisting mainly of $\mathrm{CD} 4^{+}$cells. Animal models indicate a role of autoreactive T-cells. Transfer of anti-GBM antibodies alone can induce disease but always with a mild glomerulonephritis. Furthermore, immunization with a short peptide, i.e. a T-cell epitope, can induce florid glomerulonephritis without measurable levels of anti-GBM antibodies [18, 19]. 
The origin of the anti-GBM antibodies is still not known but interestingly naturally occurring anti-GBM antibodies of IgG class have been found in all healthy individuals. These antibodies have low affinity and low titres but are specifically directed against the same epitopes found in patients with anti-GBM disease [20].

\subsection{Genetics}

Genetic studies have revealed a strong link to exist between anti-GBM disease and HLA-DRB1*1501 and DRB1*1502. Most reports stem from Caucasian populations where the DRB1-15 antigen is found in $70-80 \%$ of patients, compared to $20-30 \%$ of the controls. A recent report from China indicates a linkage also among Asians [21]. A negative link is found to HLA-DR7 and DR1, thus acting protective [22].

\subsection{Epidemiology}

Published patient series have come from New Zealand, Australia, UK, the US, China, and Scandinavia and estimated frequencies varies from $0,5-1$ cases per million inhabitants per year [7, 23, 24]. No major differences between Asian and Caucasian populations are found, as seen in many other diseases. There are two peaks of agedependent incidence, in the third and in the seventh decades. The disease is uncommon before puberty and the male to female ratio is about equal in modern series from Caucasians, but in older series and in reports from China males dominate [7, 22, 25].

\subsection{Environmental agents}

Several attempts have been made to find an association with viral infections but only anecdotal case reports have been published. Some reports describe the development of anti-GBM disease after lithotripsy treatment for renal stones, but this association was not confirmed in a larger study [26]. Exposure to chemical agents, such as organic 
solvents and cigarette smoke has also been proposed to be implicated. Smoking seems at least to predispose for lung manifestations of the disease has [27]. However there is no evidence that any of these factors can induce disease alone, although it is likely that all of them can turn an on-going subacute disease into an acute one. The limited epitope recognition is compatible with a potential role for molecular mimicry or selfimmunization with fragments of the antigen.

\section{Clinical aspects}

\subsection{Clinical manifestations}

The typical presentation is that of a reno-pulmonary syndrome i.e. the combination of renal and pulmonary insufficiency. However, many other types of presentations have been described (Table 1). Most patients report some form of general prodromal symptoms such as fatigue and malaise but usually only confined to the preceding few weeks or months. In some series, more than $50 \%$, of the patients present with only renal involvement. Virtually all have microscopic haematuria, many also have macroscopic haematuria and rapidly progressive renal insufficiency is common. Sometimes the progression is explosive leading to anuria within days, while a minority of cases experience a protracted course where the renal function is preserved for several months. Proteinuria is usually modest but occasionally patients present with a nephrotic syndrome.

The symptoms of pulmonary involvement include cough, shortness of breath, haemoptysis, chest pain and hypoxia, but patients can be asymptomatic[28]. Alveolar haemorrhage often results in anaemia, which can be severe. Chest radiography typically shows extensive bilateral air-space consolidation. This consolidation usually resolves within 2-3 days and is replaced by a reticulonodular pattern and interlobular septal 
thickening [29]. Findings at chest radiography may occasionally be normal, despite the presence of diffuse pulmonary haemorrhage. When using broncoalveolar lavage as gold standard Lazor et al found the sensitivity to be $86 \%$ for chest X-ray, and results for high resolution computed tomography were not better (80\%) [28].

Even though the underlying pathogenic mechanisms are the same the relationship between renal and pulmonary disease varies between patients both regarding severity and regarding temporal aspects. Pulmonary symptoms may start weeks to months before renal disease is diagnosed, but pulmonary symptoms may also commence first after patients have begun dialysis treatment [28]. A small minority of patients are diagnosed with normal renal function despite severe alveolar haemorrhage [30]. Lê Quang et al reviewed 29 cases described in the literature [31], many of these patients had mild urinary abnormalities. In renal disease, severity and prognosis have been shown to correlate to levels of circulating anti-GBM; such a relationship has never been found in pulmonary disease $[7,10]$.

\subsection{Pathological features}

Light microscopy typically reveals general widespread crescent formation. The percentage of glomeruli exhibiting crescents often exceeds $80 \%$, and the percentage usually correlates to renal function as well as outcome after treatment. Sometimes the light microscopy resembles the findings in Membranous Nephropathy; such patients usually present with heavy proteinuria [32]. The typical finding in direct immunofluorescence microscopy is a linear staining of $\operatorname{IgG}$ along the GBM, often accompanied by $\mathrm{C} 3$ deposition. Other staining patterns are sometimes seen, especially in mild cases with preserved renal function as well as in severely damaged glomeruli (table 2). 


\subsection{Serological features}

Anti-GBM antibodies are per definition present in all patients with anti-GBM / Goodpasture's disease. Circulating anti-GBM antibodies can be detected with indirect IF, western blotting or ELISA. In indirect IF, serum from the patient is overlaid a section of normal kidney. A good substrate and a good pathologist are needed since unspecific staining can be difficult to distinguish from the true linear staining pattern. Low levels of circulating autoantibodies can usually not be detected with indirect IF. Many labs have their in house anti-GBM assay or western blotting methodology, and there are several commercially available ELISA kits on the market. The performances of these assays depend on the purity of the antigen preparation, but are generally good [33]. Tissue bound anti-GBM antibodies can be visualized by direct immunofluorescence of renal biopsy specimens (figure 1). In patients presenting as reno-pulmonary syndromes anti-GBM antibodies can usually be found in around one third of the cases, in patients with rapidly progressive glomerulonephritis without pulmonary symptoms less than 5\% have circulating anti-GBM.

Many patients (20-35\%) with anti-GBM antibodies also have anti-neutrophil cytoplasmic antibodies (ANCA), mostly with specificity for myeloperoxidase (MPOANCA). Some double-positive patients have features typical for granulomatosis with polyangiitis or microspopic polyangitis, but virtually all published cases have severe renal disease $[34,35]$. It is therefore recommended that ANCA and anti-GBM should be analysed in parallel in patients with renal disease. (table 3)

\subsection{Diagnostic criteria}

The diagnosis of anti-GBM disease relies on the detection of anti-GBM antibodies in conjunction with glomerulonephritis and/or alveolitis. As described above there is a 
huge variation in severity of both renal and pulmonary engagement in anti-GBM disease. The diagnosis can be made also in patients with mild disease in both organs, even though this is uncommon in clinical practice. The symptoms are problematic to interpret only when features of other diseases simultaneously are present.

In most cases anti-GBM antibodies can be detected both in the circulation by serological methods (i.e. ELISA) and in the kidney by immunostaining of renal biopsies. However, sometimes these assays yield divergent results making the diagnosis more difficult. Several cases have been described where no circulating antibodies could be detected. There are several possible explanations for such findings. Immunostaining can give false positive results and it is well known that in for instance diabetes a nonspecific binding of IgG along the GBM can occur, which can be misinterpreted as antiGBM disease. The half-life of circulating antibodies is much shorter (21 days) than the half-life of tissue bound antibodies (several months). Subsequently if the sample for diagnosis is taken after the autoantibody production has ceased the assay for circulating antibodies can be true negative. There are also reports of false negative testing, reasons for this can be autoantibodies reacting with other antigens or other epitopes than the usual anti- $\alpha 3(\mathrm{IV}) \mathrm{NC} 1$. We have recently reported on anti-GBM with predominance of IgG4 that reacted better with antigen coated during non-denaturing conditions [36]. There are also reports of IgA anti-GBM reacting with other antigens.

Occasionally there are situations when tests for circulating antibodies are positive while immunostaining is negative or inconclusive. In cases with severe destruction of glomeruli the typical linear fluorescence can be difficult to identify, and in the coexistence of other autoimmune processes other depositions of immunoglobulin and/or complement components may blur the picture. It is not clear if circulating antibodies 
precede kidney bound antibodies, but it is evident that circulating antibodies precede clinical disease. If antibodies detected before disease onset should be considered as false positive or not is a philosophical questions. No doubt there is also true false positive testing. This can be due to autoantibodies reacting with other antigens than $\alpha 3$ (IV)NC1, and about $1 \%$ of sera sent for autoantibody testing contain unspecific reactivity.

\subsection{Treatment and prognosis}

The standard therapy today consists of the combination of high doses of

Kommentar [MS1]: Här behöver vi diskutera rubriker och var det ska stå om serologiska fynd.

Kommentar [TH2]: Om vi skriver klart denna meningen så tycker jag att det är ok. Jag har tagit bort diabetes från stycket ovan. Annars finns det en logic I det som repeteras. glucocorticoids, cyclophosphamide and plasma-exchange. The rational with steroids is to halt neutrophil driven inflammation; with cyclophosphamide to stop autoantibody production and with plasma exchange to remove circulating autoantibodies. Such therapy is usually very successful if started early. If begun before s-creatinine has risen above 600 micromole/L ( $5 \mathrm{mg} / \mathrm{dl})$ there is a good chance of renal survival [7, 37]. Even if the chance for renal recovery is small when treatment is started later, there are dialysis dependent cases that do recover. Considering that there is a risk for late onset of alveolar haemorrhage it may be unwise not to treat all patients. Patients should not be transplanted as long as circulating autoantibodies can be detected. When anti-GBM production has ceased, it is very rare with relapses; we found one case in 81 while Levy et al found 2 among $71[7,37]$.

\section{Concluding remarks}

The clinical expression can vary but anti-GBM disease in man has a poor prognosis. The rapidly progressive glomerulonephritis combined with lung haemorrhage often result in death of the patient if not treated. Results obtained with combined immunosuppression and plasma exchange have shown a much-improved prognosis, although it is still important to initiate therapy before renal damage has advanced too 
far. Early recognition of cases is therefore mandatory and can be obtained by the use of sensitive assays. Anti-GBM disease has been thoroughly studied during the last 50 years and can be considered a model of autoimmune diseases in general. The disease can be transferred with the antibodies. The antigen is well known as the C-terminal end of the alpha-3 chain of type IV collagen and the cryptic epitope is well defined. Models of anti-GBM nephritis resembling of Goodpasture's disease is today one of the most widespread models used to study inflammatory processes. 


\section{Tables}

Table 1. Symptoms and signs of Goodpasture's disease at presentation

\begin{tabular}{lll} 
Signs and symptoms & Prevalence & Comments \\
\hline Rapidly progressive GN & $99 \%$ & \\
Lung involvement & $30-60 \%$ & \\
Malaise, fatigue and weight loss & $70-90 \%$ & Related to anaemia, uraemia or degree of \\
& & inflammation \\
Arthralgia and myalgia & Rare & May not be related to Goodpasture's disease \\
Hypertension & & Late event due to advanced renal failure with fluid \\
& & retention
\end{tabular}


Table 2. Laboratory findings

\begin{tabular}{|c|c|c|}
\hline Method & Finding & Comment \\
\hline Light microscopy of renal & Extensive crescent formation, & \\
\hline \multirow[t]{5}{*}{ tissue } & diffuse proliferative & \\
\hline & glomerulonephritis with variable & \\
\hline & degree of necrosis, & \\
\hline & glomerulosclerosis and tubular & \\
\hline & loss & \\
\hline \multirow[t]{5}{*}{ IF on renal tissue } & Linear binding of IgG along the & Can occasionally be seen in SLE, diabetes \\
\hline & glomerular basement membrane & mellitus, renal transplants and in some \\
\hline & & normal kidneys \\
\hline & C3 deposition & Found in about $75 \%$ of cases \\
\hline & $\operatorname{IgA}$ or IgM & Found in $10-15 \%$ \\
\hline Urine analysis & Haematuria, proteinuria & \\
\hline s-Creatinine & Elevated & \\
\hline
\end{tabular}


Table 3. Serological findings

Serological feature

Prevalence

Anti-GBM

$100 \%$

ANCA (mostly MPO-ANCA)

$20-35 \%$ 


\section{Figure legends}

Figure 1. Direct IF of human kidney biopsy. IgG is visualized and a linear staining is found along the GBM. This picture is identical to staining using anti- $\alpha 3$ (IV) monoclonal antibodies.

Figure 2. A) The type IV collagen network is building up the scaffold of basement membranes. Each collagen molecule is comprised of three of $\alpha(\mathrm{IV})$ chains and in human GBM only molecules with one $\alpha 3$ (IV), one $\alpha 4(\mathrm{IV})$ and one $\alpha 5$ (IV) chain is found. Four collagen IV molecules are connected in the N-terminal end. B) Two collagen IV molecules are connected via their C-terminal ends, in which each $\alpha(I V)$ chain is folded into a globular domain, the NC1 domain. C) Shows a model of the NC1 hexamer of type IV collagen found in the GBM. Each type IV collagen molecule is composed of one $\alpha 3$, one $\alpha 4$ and one $\alpha 5$ chain. The two $\alpha 4 \mathrm{NC} 1$ domains bind to each other whereas the $\alpha 3$ binds an $\alpha 5 \mathrm{NC} 1$ domain. The amino acids identified as the epitope of the pathogenic antibodies is indicated on one of the $\alpha 3$ molecules in white and the large arrow. The proposed positions of the six $\alpha(\mathrm{IV}) \mathrm{NC} 1$ domains found in the human GBM are indicated. Note that the two $\alpha 4(\mathrm{IV})$ domains are positioned on the back of the molecule. This picture of the NC1 hexamer is modelled from the NCBI MMDB entry \#29412 using the Cn3D software (NCBI). 


\section{References}

[1] Jennette JC, Falk RJ, Bacon PA, Basu N, Cid MC, Ferrario F et al. 2012 revised International Chapel Hill Consensus Conference Nomenclature of Vasculitides. Arthritis Rheum, 2013;65:1-11.

[2] Stanton MC, Tange JD. Goodpasture's syndrome (pulmonarry haemorrhage associated with glomerulonephritis). AustAnnMed, 1958;7:132-44.

[3] Goodpasture EW. The significance of certain pulmonary lesions in relations in relation to the etiology of influenza. AmJMedSci, 1919;158:863-70.

[4] Lerner RA, Glassock RJ, Dixon FJ. The role of anti-glomerular basement membrane antibody in the pathogenesis of human glomerulonephritis. JExpMed, 1967;126:9891004 .

[5] Wieslander J, Bygren P, Heinegard D. Isolation of the specific glomerular basement membrane antigen involved in Goodpasture syndrome. Proc Natl Acad Sci U S A, 1984;81:1544-8.

[6] Kleppel MM, Santi PA, Cameron JD, Wieslander J, Michael AF. Human tissue distribution of novel basement membrane collagen. The American journal of pathology, 1989;134:813-25.

[7] Segelmark M, Hellmark T, Wieslander J. The prognostic significance in Goodpasture's disease of specificity, titre and affinity of anti-glomerular-basement-membrane antibodies. Nephron Clinical practice, 2003;94:c59-68.

[8] Cui Z, Zhao MH, Xin G, Wang HY. Characteristics and prognosis of Chinese patients with anti-glomerular basement membrane disease. Nephron Clinical practice, 2005;99:c49-55.

[9] Segelmark M, Dahlberg P, Wieslander J. Anti-GBM disease with a mild relapsing course and low levels of anti-GBM autoantibodies. Clinical Kidney Journal, 2012;5:549-51.

[10] Yang R, Hellmark T, Zhao J, Cui Z, Segelmark M, Zhao MH et al. Levels of epitopespecific autoantibodies correlate with renal damage in anti-GBM disease. Nephrology, dialysis, transplantation : official publication of the European Dialysis and Transplant Association - European Renal Association, 2009;24:1838-44.

[11] Wieslander J, Langeveld J, Butkowski R, Jodlowski M, Noelken M, Hudson BG. Physical and immunochemical studies of the globular domain of type IV collagen. Cryptic properties of the Goodpasture antigen. J Biol Chem, 1985;260:8564-70.

[12] Hellmark T, Johansson C, Wieslander J. Characterization of anti-GBM antibodies involved in Goodpasture's syndrome. Kidney international, 1994;46:823-9.

[13] Hellmark T, Segelmark M, Unger C, Burkhardt H, Saus J, Wieslander J. Identification of a clinically relevant immunodominant region of collagen IV in Goodpasture disease. Kidney international, 1999;55:936-44. 
[14] Hudson BG, Tryggvason K, Sundaramoorthy M, Neilson EG. Alport's syndrome, Goodpasture's syndrome, and type IV collagen. N Engl J Med, 2003;348:2543-56.

[15] Hellmark T, Burkhardt H, Wieslander J. Goodpasture disease. Characterization of a single conformational epitope as the target of pathogenic autoantibodies. J Biol Chem, 1999;274:25862-8.

[16] Borza DB, Bondar O, Colon S, Todd P, Sado Y, Neilson EG et al. Goodpasture autoantibodies unmask cryptic epitopes by selectively dissociating autoantigen complexes lacking structural reinforcement: novel mechanisms for immune privilege and autoimmune pathogenesis. J Biol Chem, 2005;280:27147-54.

[17] Vanacore RM, Ham AJ, Cartailler JP, Sundaramoorthy M, Todd P, Pedchenko V et al. A role for collagen IV cross-links in conferring immune privilege to the Goodpasture autoantigen: structural basis for the crypticity of B cell epitopes. J Biol Chem, 2008;283:22737-48.

[18] Lou YH. Anti-GBM glomerulonephritis: a T cell-mediated autoimmune disease? Arch Immunol Ther Exp (Warsz), 2004;52:96-103.

[19] Bolton WK, Chen L, Hellmark T, Wieslander J, Fox JW. Epitope spreading and autoimmune glomerulonephritis in rats induced by a T cell epitope of Goodpasture's antigen. Journal of the American Society of Nephrology : JASN, 2005;16:2657-66.

[20] Yang R, Cui Z, Hellmark T, Segelmark M, Zhao MH, Wang HY. Natural anti-GBM antibodies from normal human sera recognize alpha3(IV)NC1 restrictively and recognize the same epitopes as anti-GBM antibodies from patients with anti-GBM disease. Clin Immunol, 2007;124:207-12.

[21] Luo H, Chen M, Cui Z, Yang R, Xu PC, Zhou XJ et al. The association of HLA-DQB1, DQA1 and -DPB1 alleles with anti- glomerular basement membrane (GBM) disease in Chinese patients. BMC nephrology, 2011;12:21.

[22] Salama AD, Levy JB, Lightstone L, Pusey CD. Goodpasture's disease. Lancet, 2001;358:917-20.

[23] Taylor DM, Yehia M, Simpson IJ, Thein H, Chang Y, de Zoysa JR. Anti-glomerular basement membrane disease in Auckland. Intern Med J, 2012;42:672-6.

[24] Savage CO, Pusey CD, Bowman C, Rees AJ, Lockwood CM. Antiglomerular basement membrane antibody mediated disease in the British Isles 1980-4. Br Med J (Clin Res Ed), 1986;292:301-4.

[25] Cui Z, Zhao MH, Xin G, Wang HY. Characteristics and prognosis of Chinese patients with anti-glomerular basement membrane disease. Nephron Clin Pract, 2005;99:c49-55.

[26] Westman KW, Ericsson UB, Hoier-Madsen M, Wieslander J, Lindstedt E, Bygren PG et al. Prevalence of autoantibodies associated with glomerulonephritis, unaffected after extracorporeal shock wave lithotripsy for renal calculi, in a three-year follow-up. Scand J Urol Nephrol, 1997;31:463-7. 
[27] Donaghy M, Rees AJ. Cigarette smoking and lung haemorrhage in glomerulonephritis caused by autoantibodies to glomerular basement membrane. Lancet, 1983;2:1390-3.

[28] Lazor R, Bigay-Game L, Cottin V, Cadranel J, Decaux O, Fellrath JM et al. Alveolar hemorrhage in anti-basement membrane antibody disease: a series of 28 cases. Medicine (Baltimore), 2007;86:181-93.

[29] Bowley NB, Steiner RE, Chin WS. The chest X-ray in antiglomerular basement membrane antibody disease (Goodpasture's syndrome). Clin Radiol, 1979;30:419-29.

[30] Harrity P, Gilbert-Barness E, Cabalka A, Hong R, Zimmerman J. Isolated pulmonary Goodpasture syndrome. Pediatr Pathol, 1991;11:635-46.

[31] Le Quang C, Delevaux I, Trouillier S, Andre M, Souweine B, Tridon A et al. [Intraalveolar haemorrhage without renal damage as the initial presenting feature of Goodpasture's syndrome: case report and review of literature]. Rev Med Interne, 2008;29:1038-42.

[32] Jia X-y, Hu S-y, Chen J-1, Qu Z, Liu G, Cui Z et al. The clinical and immunological features of patients with combined anti-GBM disease and membranous nephropathy. Kidney international, 2013;In press.

[33] Sinico RA, Radice A, Corace C, Sabadini E, Bollini B. Anti-glomerular basement membrane antibodies in the diagnosis of Goodpasture syndrome: a comparison of different assays. Nephrol Dial Transplant, 2006;21:397-401.

[34] Hellmark T, Niles JL, Collins AB, McCluskey RT, Brunmark C. Comparison of antiGBM antibodies in sera with or without ANCA. Journal of the American Society of Nephrology : JASN, 1997;8:376-85.

[35] Chen M, Cui Z, Zhao MH. ANCA-associated vasculitis and anti-GBM disease: the experience in China. Nephrol Dial Transplant, 2010;25:2062-5.

[36] Ohlsson S, Herlitz H, Lundberg S, Selga D, Mölne J, Wieslander J et al. Circulating AntiGlomerular Basement Membrane Antibodies with Predominance of Subclass IgG4 and False Negative Immunoassay Test Results in Anti-GBM Disease. Am J Kidney Dis, 2013; In press.

[37] Levy JB, Turner AN, Rees AJ, Pusey CD. Long-term outcome of anti-glomerular basement membrane antibody disease treated with plasma exchange and immunosuppression. Ann Intern Med, 2001;134:1033-42. 